$\underline{\text { Supporting Information }}$

\title{
Monte Carlo Simulations of Nanopore Compartmentalization Yield Fingerprint Adsorption Isotherms as a Rationale for Advanced Structure Characterization of Metal-Organic Frameworks
}

\author{
Shivam Parashar $^{\dagger}$, Qing Zhu ${ }^{\dagger}{ }^{\dagger}$, Silvio Dantas ${ }^{\dagger}$, Alexander V. Neimark ${ }^{\dagger}, *$ \\ $\dagger^{\dagger}$ Department of Chemical and Biochemical Engineering, Rutgers, The State University of New Jersey, Piscataway, \\ New Jersey 08854, United States \\ tDepartment of Physics, Applied Physics, and Astronomy - Rensselaer Polytechnic Institute, Troy, New York \\ 12180, United States \\ *E-mail: aneimark@rutgers.edu
}

A. Simulation Method

Table S1. LJ parameters used for performing Grand Canonical Monte Carlo (GCMC) simulations.

\begin{tabular}{|c|c|c|c|c|}
\hline & Atom types & $\sigma(\AA)$ & $\varepsilon / \mathbf{k}_{\mathrm{B}}(\mathbf{K})$ & reference \\
\hline \multirow[t]{6}{*}{ Framework } & C & 3.47 & 47.86 & Dreiding ${ }^{1}$ \\
\hline & $\mathrm{H}$ & 2.85 & 7.65 & Dreiding 1 \\
\hline & $\mathrm{O}$ & 3.03 & 48.16 & Dreiding 1 \\
\hline & $\mathrm{N}$ & 3.26 & 38.95 & Dreiding 1 \\
\hline & $\mathrm{Zr}$ & 2.78 & 34.72 & $\mathrm{UFF}^{2}$ \\
\hline & $\mathrm{Zn}$ & 2.46 & 62.42 & $\mathrm{UFF}^{2}$ \\
\hline \multirow[t]{4}{*}{ Adsorbate } & $\mathrm{Ar}$ & 3.4 & 119.8 & ref. [3] \\
\hline & $\mathrm{N} \_\mathrm{N}_{2}$ & 3.31 & 36 & TraPPE ${ }^{4}$ \\
\hline & $\mathrm{C} \_\mathrm{CO}_{2}$ & 2.8 & 27 & TraPPE $^{4}$ \\
\hline & $\mathrm{O} \mathrm{CO}_{2}$ & 3.05 & 79 & TraPPE $^{4}$ \\
\hline
\end{tabular}

Table S1 summarizes the Lennard-Jones parameters for the framework and adsorbate atoms used for performing grand canonical Monte Carlo and Gibbs ensemble simulations to determine adsorption isotherms and saturation pressures (Table S2), respectively. For the Gibbs ensemble simulations, the probabilities for the adsorbate translation, rotation, swap, reinsertion moves were set to 0.244 and simulation cell volume change to 0.024 . The visualization was done using VMD 
1.9.35 and all plots were made using python module matplotlib.

Table S2. LJ potential truncation schemes used in GCMC simulation. Saturation pressures of Ar and $\mathrm{N}_{2}$ $(77.4 \mathrm{~K}$ and $87.3 \mathrm{~K})$ were computed using the Gibbs ensemble simulations. Saturation pressure of $\mathrm{CO}_{2}$ (194.7 K) was assumed to be $1 \mathrm{~atm}$.

\begin{tabular}{ccccc}
\hline Adsorbates & $\begin{array}{c}\text { Shifted or } \\
\text { Truncated }\end{array}$ & Tail corrections & Cutoff $(\AA)$ & P $_{\text {sat }}(\mathbf{a t m})$ \\
\hline $\mathrm{Ar}$ & Truncated & No & 17 & 0.918 \\
\hline $\mathrm{N}_{2}$ & Shifted & No & 17 & 1.252 \\
\hline $\mathrm{CO}_{2}$ & Shifted & No & $\begin{array}{c}12 \text { for PCN-224 and } \\
15 \text { for ZIF-412 }\end{array}$ & 1.000 \\
& & & \\
\hline
\end{tabular}

\section{Polarization in $\mathrm{CO}_{2}$ adsorption on $\mathrm{PCN}-224$}

A study conducted on $\mathrm{CO}_{2}$ adsorption in MgMOF-74 revealed that UFF underpredicted the $\mathrm{CO}_{2}$ adsorption at low pressure compared to experiments. ${ }^{6}$ But when polarization is explicitly considered, the agreement becomes better with experiments. Similar to MgMOF-74, GCMC simulations without polarization also underpredict $\mathrm{CO}_{2}$ adsorption on $\mathrm{PCN}-224$. This motivated us to include polarization effects into our simulations. Isotherms with and without polarization are shown in Figure S1. The Polarizabilities were taken from ref. 7 and scaled by 0.03 (see Table S3). The scaling factor of 0.03 was chosen by fitting the simulated isotherm at low pressures to experiments.

Table S3. Polarizabilities of framework atoms and adsorbate atoms used for $\mathrm{CO}_{2}$ adsorption simulations.

\begin{tabular}{ccc}
\hline & Atom types & $\boldsymbol{\alpha}\left(\AA^{3}\right)$ \\
\hline Framework & $\mathrm{C}$ & 0.305 \\
$\mathrm{H}$ & 0.105 \\
$\mathrm{O}$ & 0.192 \\
$\mathrm{~N}$ & 0.228 \\
$\mathrm{Zr}$ & 0.079 \\
$\mathrm{Zn}$ & 0.124 \\
\hline Adsorbate & $\mathrm{C}_{-} \mathrm{CO}_{2}$ & 0.305 \\
& $\mathrm{O}_{-} \mathrm{CO}_{2}$ & 0.192
\end{tabular}




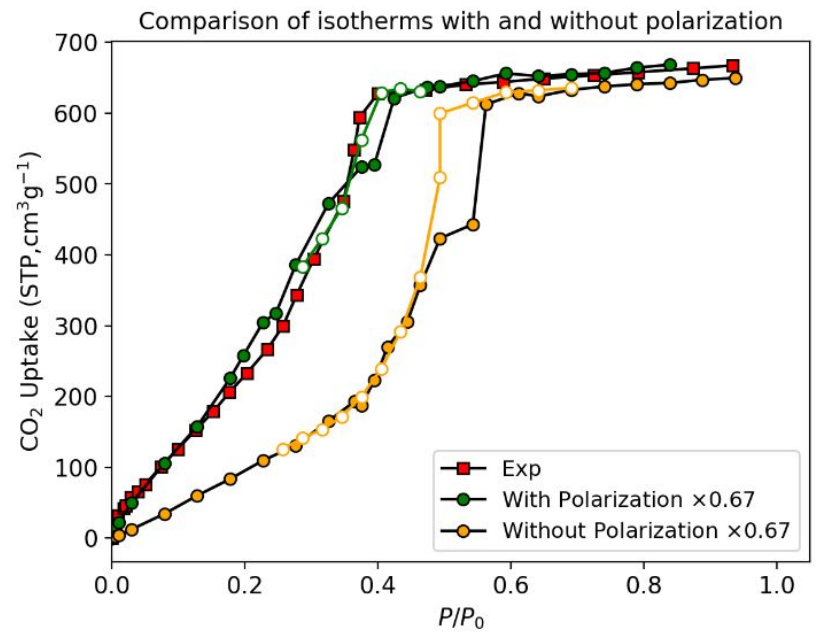

Figure S1: Effect of adsorbate-framework polarization on $\mathrm{CO}_{2}$ adsorption isotherm on $\mathrm{PCN}-224$. The simulated isotherms (green and orange) were scaled by a factor of 0.67 .

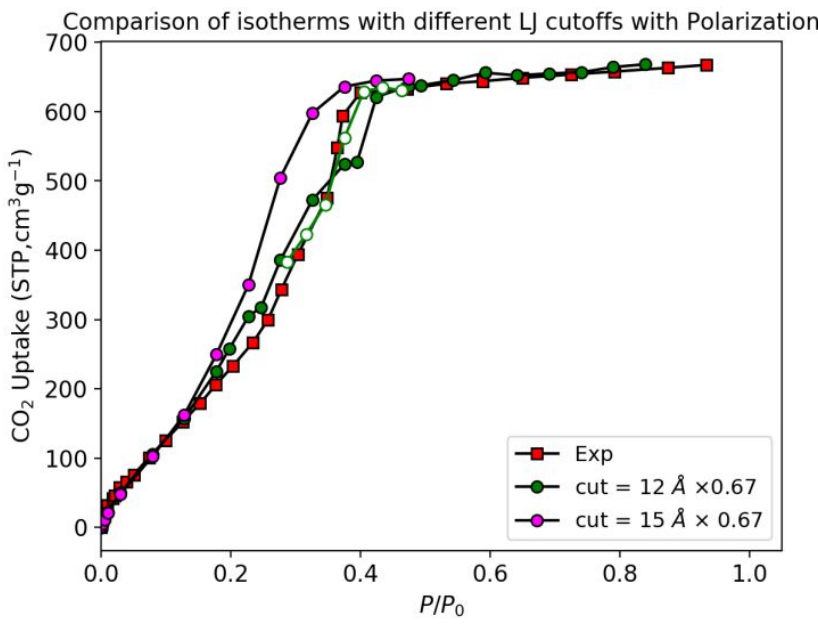

Figure S2: Effect of LJ potential cutoff on $\mathrm{CO}_{2}$ adsorption isotherm on PCN-224. The simulated isotherms (green and magenta) were scaled by a factor of 0.67 .

\section{Density Profile Calculation}

The density profile (Figure 4 and Figure 10 of main text) was computed by considering a cylindrical differential volume (Figure S3) along the given direction. This volume was split into 
bins and density was computed by dividing the average number of molecules in each bin by the volume of differential volume element. The radius of the cylinder was chosen to be $2.5 \AA$ for PCN224 and $4 \AA$ for ZIF-412.

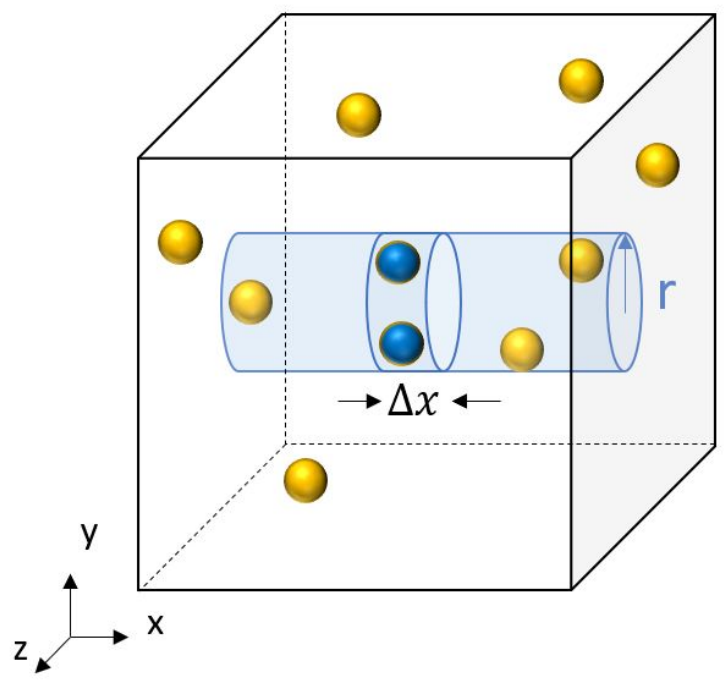

Figure S3: Schematic of density profile calculation along a given direction. Cylindrical volume was chosen to calculate average number of molecules along given direction.

\section{B. Dependence on the potential truncation beyond cutoff}

Table S4: Saturation pressure of Argon and $N_{2}$ at $87.3 \mathrm{~K}$ and $77.4 \mathrm{~K}$, respectively with 17 A cutoff, using different truncation schemes.

\begin{tabular}{ccc}
\hline Saturation Pressure (atm) & $\mathbf{A r}$ & $\mathbf{N}_{\mathbf{2}}$ \\
\hline Shifted & 0.974 & 1.252 \\
\hline Truncated no tail & 0.918 & 1.120 \\
\hline Truncated with tail & 0.849 & 1.076 \\
\hline
\end{tabular}

Table S4 summarizes the saturation pressures of $\mathrm{Ar}$ and $\mathrm{N}_{2}$ depending on the type of truncation. The saturation pressures were computed using the Gibbs Ensemble simulations. To see how the choice of truncation dictates the shape of the Ar isotherm, we plot Figure S4. The condensation step of Ar on PCN-224 using shifted potential is $\sim 10 \mathrm{kPa}$ to the right of truncated 
potential with tail corrections. This shows that the adsorption isotherm depends significantly on the way of potential truncation even with a longer cutoff of $5 \sigma(17 \AA)$. The difference between the isotherms obtained with different truncation methods reduces on plotting the isotherms against the relative pressure (Figure S4 b,d). A similar comparison is shown for $\mathrm{N}_{2}$ in Figure S5.
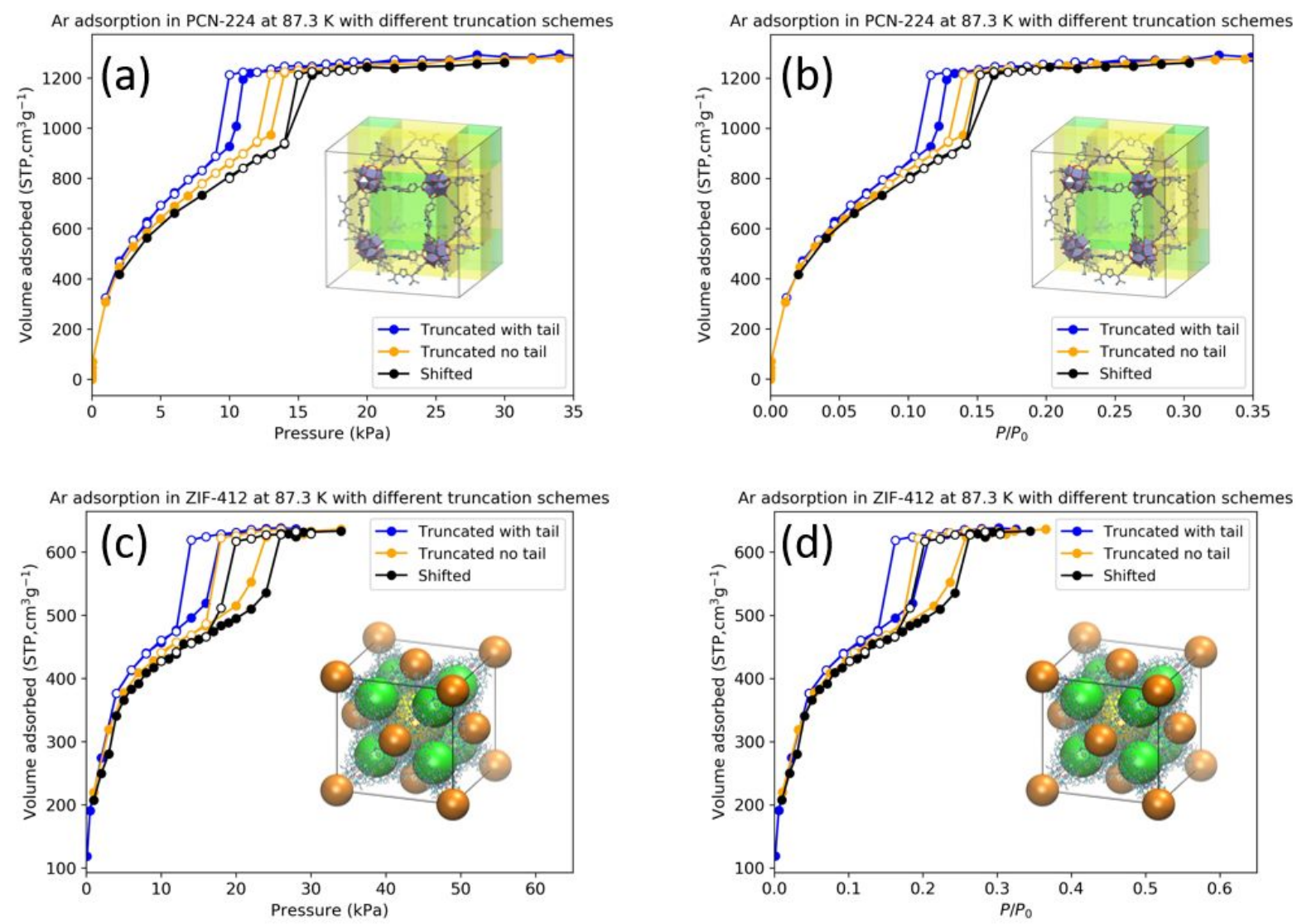

Figure S4: Comparison of Argon isotherms with different way of potential truncation beyond cutoff in $(a, c)$ absolute and $(b, d)$ relative pressure scale for PCN-224 and ZIF-412. Plots with closed and open circle are adsorption and desorption isotherms, respectively. The saturation pressures used to compute the relative pressures are summarized in Table S4. 

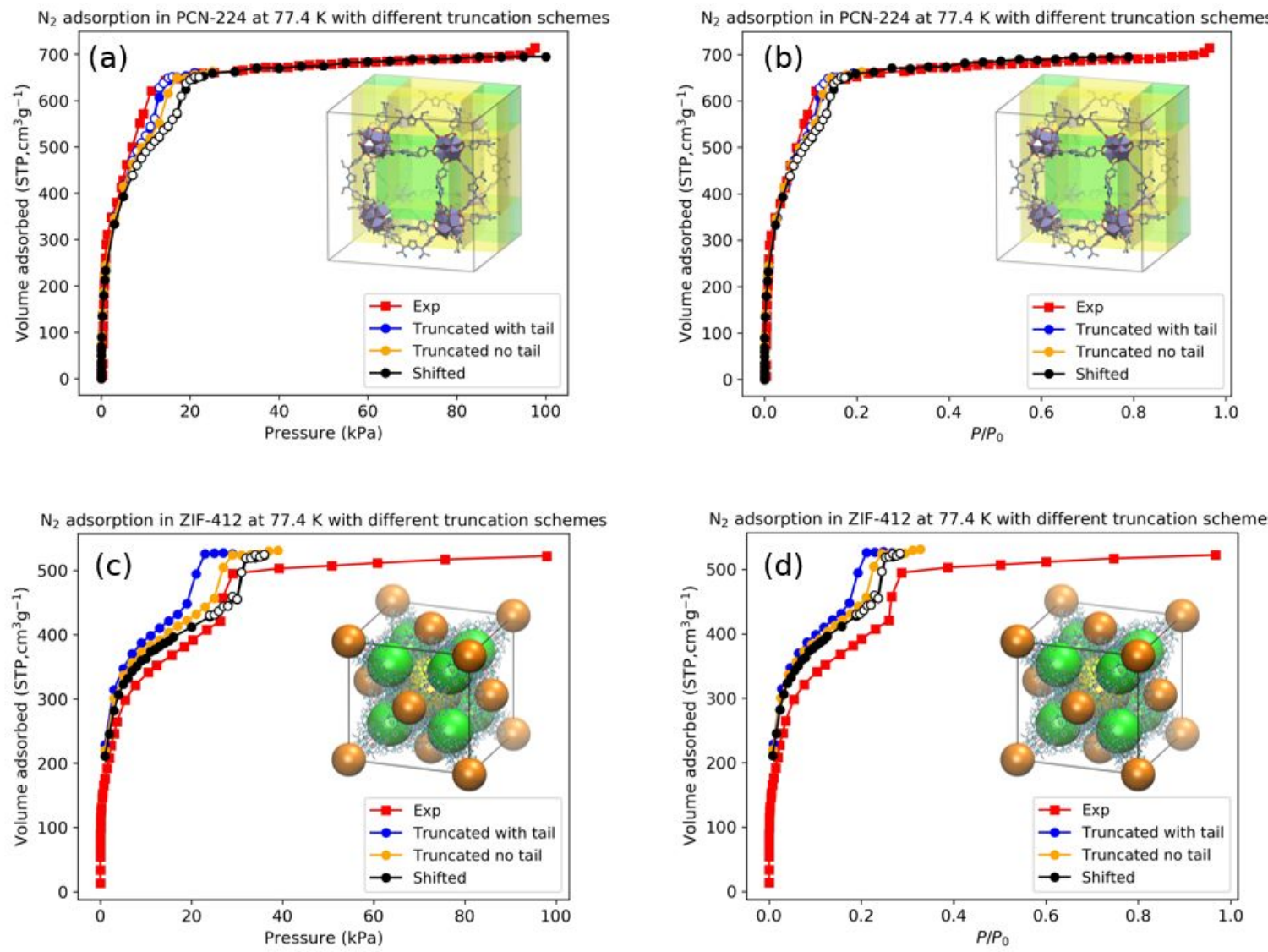

Figure S5: Comparison of $\mathrm{N}_{2}$ isotherms with different way of potential truncation beyond cutoff in (a, c) absolute and $(b, d)$ relative pressure scale for PCN-224 and ZIF-412. Plots with closed and open circle are adsorption and desorption isotherms, respectively. The saturation pressures are summarized in Table S4. Experimental isotherm is obtained from ref [8]. 


\section{Dependence on the temperature}

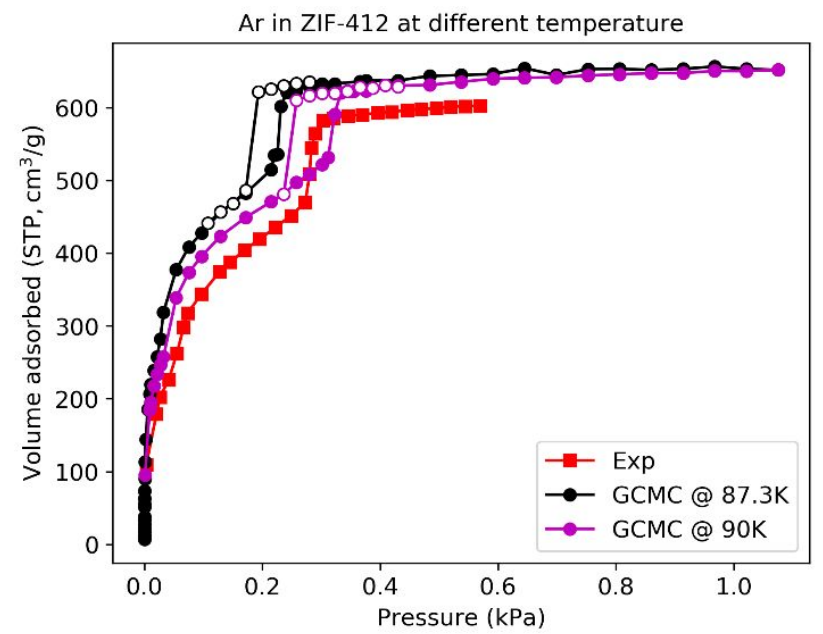

Figure S6: Experimental isotherm (red) aligns reasonably well with GCMC simulations at $89 \mathrm{~K}$. The closed and open circle are adsorption and desorption points, respectively.

\section{Pore Volumes}

Here we compare pore volumes obtained using simulations and experiments for Argon and a similar discussion follows for $\mathrm{N}_{2}$ and $\mathrm{CO}_{2}$. The simulated and experimental pore volume of Argon in PCN-224 is $1.70 \mathrm{cc} / \mathrm{g}$ and $1.01 \mathrm{cc} / \mathrm{g}$ (Table S5). They differ because the experimental sample was not fully crystalline. The percentage contribution of each type of cage are in close agreement channel/intersection pore occupy $69.4 \%$ / $30.6 \%$ of the total volume accessed by Argon in ideal PCN-224 and $68.3 \% / 31.6 \%$ in experimental sample.

The simulated pore volume of Argon in ZIF-412 is $3.33 \mathrm{cc} / \mathrm{g}$ vs $3.13 \mathrm{cc} / \mathrm{g}$ from experiments. The lta, fau and ucb pores contribute to $10.8 \%, 23.4 \%, 65.8 \%$ respectively of the total volume. This percentage contributions from each pore are slightly different from experiments: $6.1 \%$, $23.6 \%, 70.3 \%$ from lta, fau and ucb pores, respectively. This is because the fau and ucb cages were almost fully accessible in the experimental sample whereas only a fraction of lta cages (53\%) were available for Ar adsorption. 
Table S5: Comparison of simulated and experimental ${ }^{8}$ pore volume of $\mathrm{PCN}-224$ probed by $\mathrm{Ar}, \mathrm{N}_{2}$ and $\mathrm{CO}_{2}$ determined using the Gurvich rule. This is the tabulated data of the bar charts presented in Figure 11 of the main text.

\begin{tabular}{ccccccc}
\hline Adsorbate & \multicolumn{2}{c}{ Channel (cc/g) } & \multicolumn{2}{c}{ Intersection $(\mathbf{c c} / \mathbf{g})$} & \multicolumn{2}{c}{ Total (cc/g) } \\
\cline { 2 - 7 } & Sim & Exp & Sim & Exp & Sim & Exp \\
\hline $\mathbf{A r}$ & 1.18 & 0.69 & 0.52 & 0.32 & 1.70 & 1.01 \\
\hline $\mathbf{N}_{\mathbf{2}}$ & 1.22 & 0.77 & 0.48 & 0.31 & 1.70 & 1.08 \\
\hline $\mathbf{C O}_{\mathbf{2}}$ & 0.87 & 0.57 & 0.37 & 0.26 & 1.24 & 0.83 \\
\hline
\end{tabular}

Table S6: Comparison of simulated and experimental ${ }^{8}$ pore volumes of $\mathrm{ZIF}-412$ probed by $\mathrm{Ar}, \mathrm{N}_{2}$ and $\mathrm{CO}_{2}$ determined using the Gurvich rule. This is the tabulated data of the bar charts presented in Figure 11 of the main text.

\begin{tabular}{lllllllll}
\hline Adsorbate & \multicolumn{2}{l}{ Ita (cc/g) } & \multicolumn{2}{l}{ fau (cc/g) } & \multicolumn{2}{l}{ ucb (cc/g) } & \multicolumn{2}{l}{ Total (cc/g) } \\
\cline { 2 - 9 } & Sim & Exp & Sim & Exp & Sim & Exp & Sim & Exp \\
\hline $\mathbf{A r}$ & 0.36 & 0.19 & 0.78 & 0.74 & 2.19 & 2.20 & 3.33 & 3.13 \\
\hline $\mathbf{N}_{2}$ & 0.36 & 0.21 & 0.79 & 0.78 & 2.19 & 2.15 & 3.34 & 3.14 \\
\hline $\mathbf{C O}_{2}$ & 0.25 & 0.17 & 0.56 & 0.54 & 1.61 & 1.70 & 2.42 & 2.41 \\
\hline
\end{tabular}

\section{E. Partial Occupancies in ZIF-412}

Although crystals are known for their periodicity, a fraction of atoms in a real crystallographic structure may not repeat regularly over long distances. When a unit cell is constructed by spatially averaging over the crystal, the same site in two unit cells may be occupied by different atoms where each atom occupies the site, but only partially (as in ZIF-412 structure $^{9}$ ). For calculating adsorption isotherms, a structure with fully occupied atoms is required because GCMC packages 
like RASPA are unable to handle partially occupied atomic sites. For a small structure, atoms can be retained or deleted according to their partial occupancies manually. ${ }^{10}$ But ZIF-412 contain several partially occupied atoms making manually picking unfeasible. We developed an algorithm that generates a set of fully occupied atom structures by deleting/retaining the atoms according to their partial occupancies (Figure S7). All the generated structures have same number of atoms types that differ only in the relative positioning of the linkers. Since identical atom types are scattered at multiple positions within a unit cell, we choose them at random for retaining/deleting. As an example, in we show two output structures (but many others are possible) from the same partially occupied structure (Figure S11). Each of the resulting structure is equally probable, differing only in the relative positions of partially occupied linkers within the unit cell.
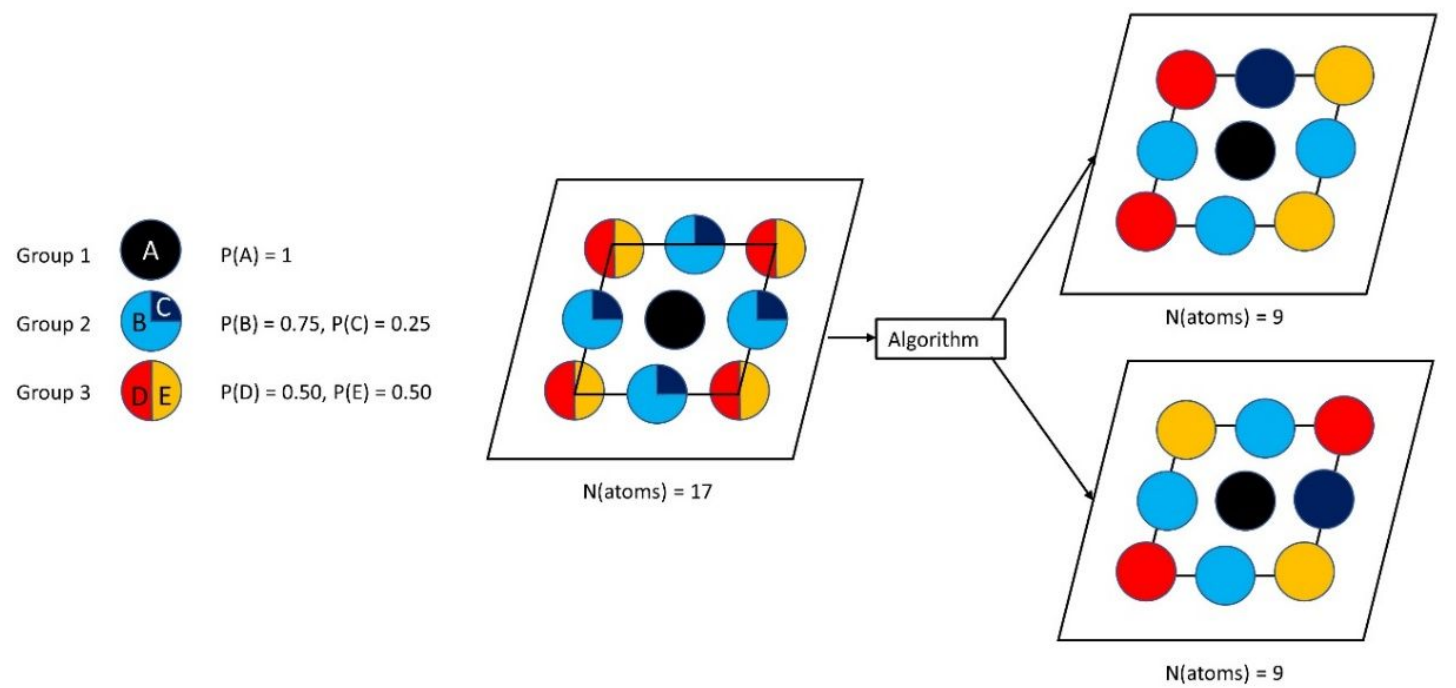

Figure S7: The problem of partial occupancy within a structure. Our algorithm takes in a partially occupied structure and returns several structures (of which two are shown) with fully occupied atoms. 


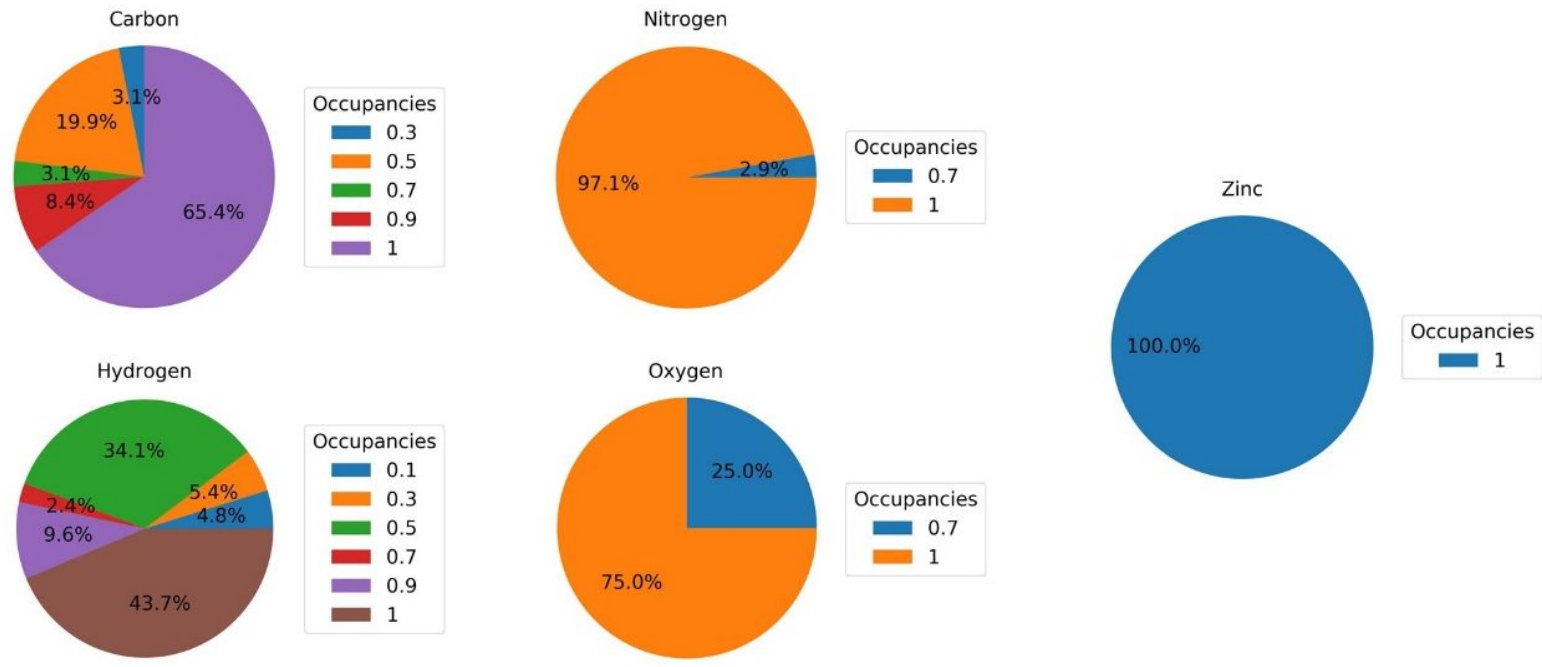

Figure S8: Percentage of partially occupied atoms in cif file obtained from ref ${ }^{9}$ for ZIF-412. All atoms of $\mathrm{Zn}$ were fully occupied. Nitrogen and Oxygen were partially occupied only in the $\mathrm{NO}_{2}$ group. Carbon and Hydrogen exhibited 5 and 6 different occupancies, respectively.

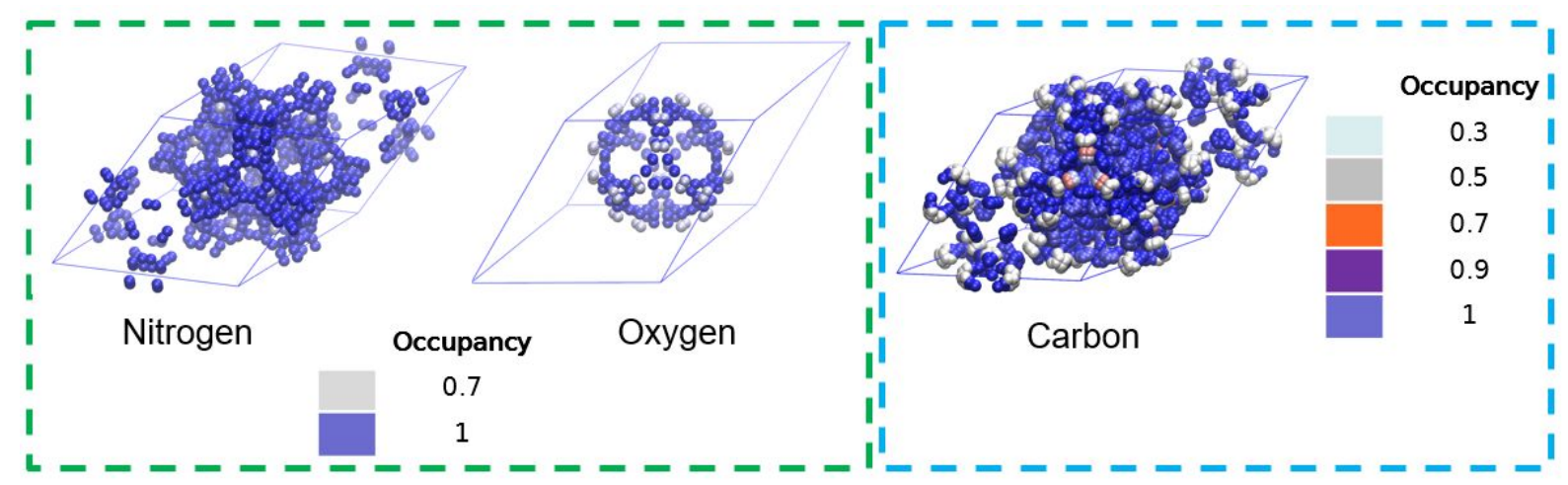

Figure S9: Location of partially occupied Nitrogen, Oxygen and Carbons within the crystallographic structure of ZIF-412. All Hydrogens connected to Carbons also exhibited the same occupancies. 


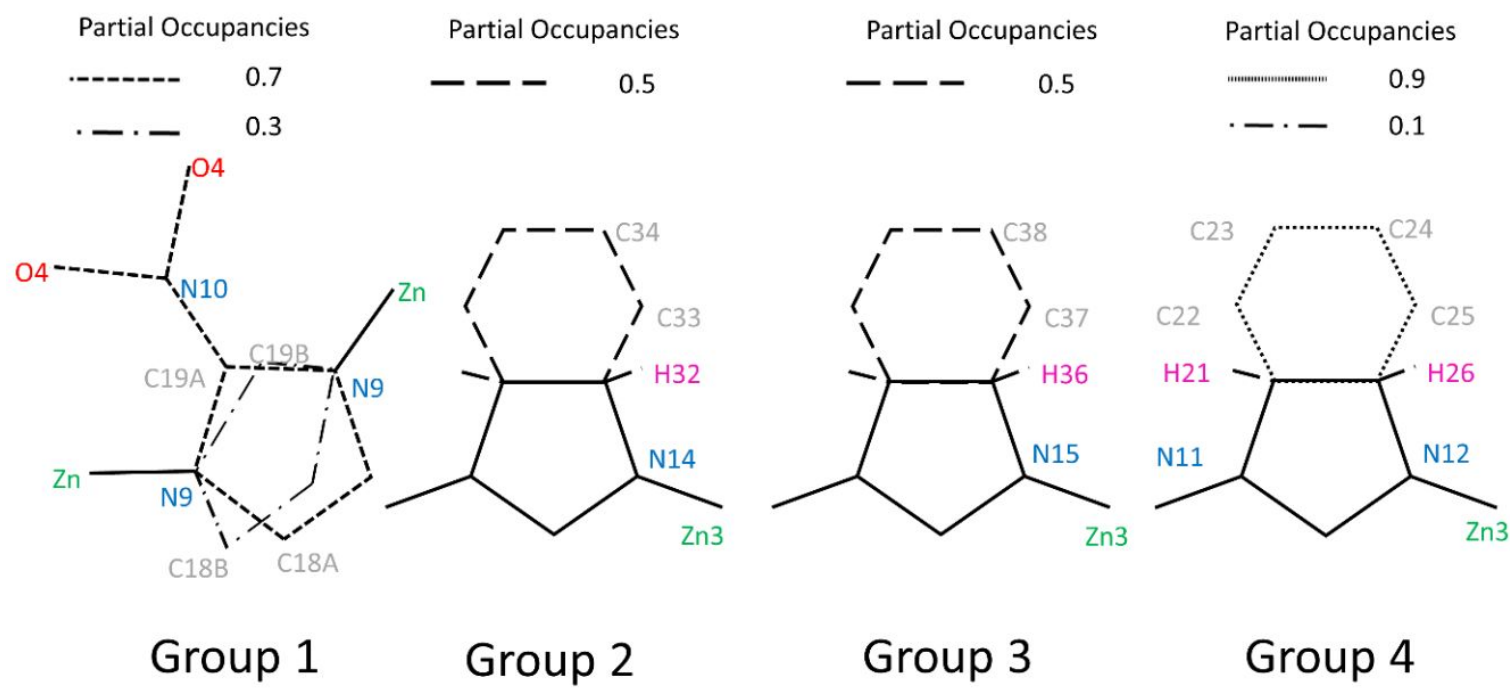

Figure S10: Partially occupied atoms were divided into 4 groups. The dashed bonds indicate that corresponding atoms are partially occupied, and their occupancy indicated in the legend of each group. Atoms connected with solid bonds had an occupancy of 1. Group 2 and 3 are similar, they differ only in the atom types. Color code for atom labels: Zinc, green; Nitrogen, blue: Carbon, gray; Oxygen, red; Hydrogen, pink.
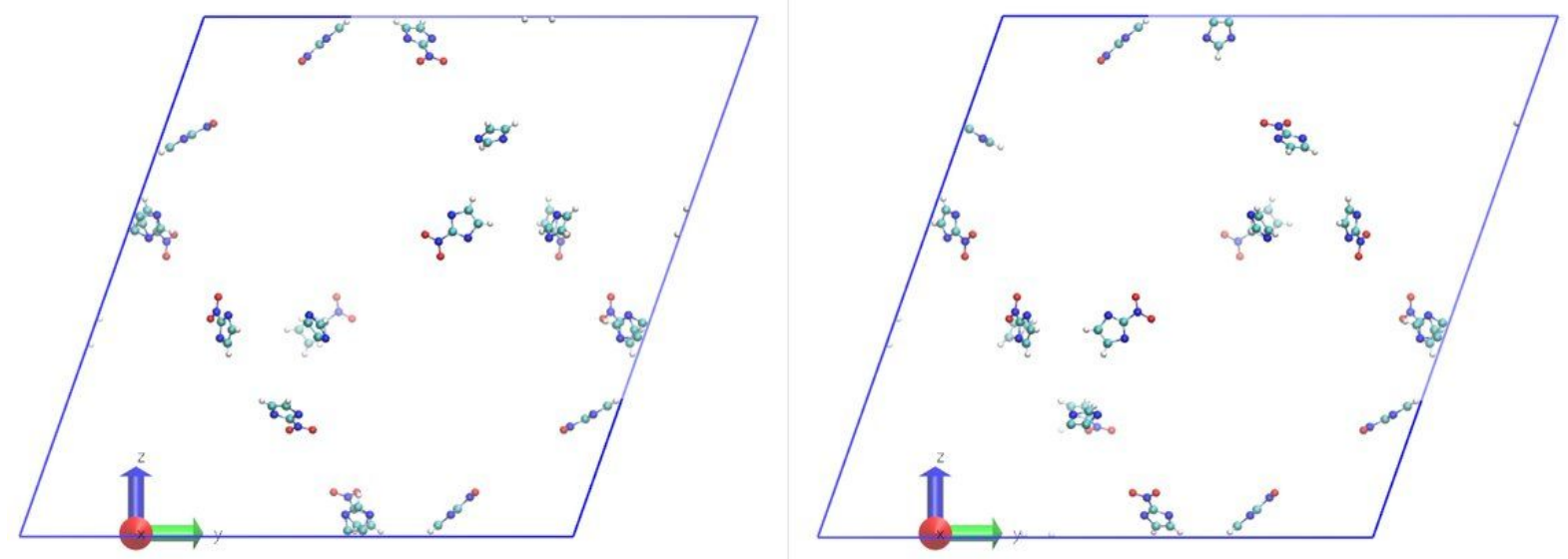

Figure S11: Comparison of two structure files generated after removing partial occupancies. Only group 4 atoms are shown for the sake of clarity. The two structure contains the same number of atoms but differ only in the relative positioning of the linkers. 


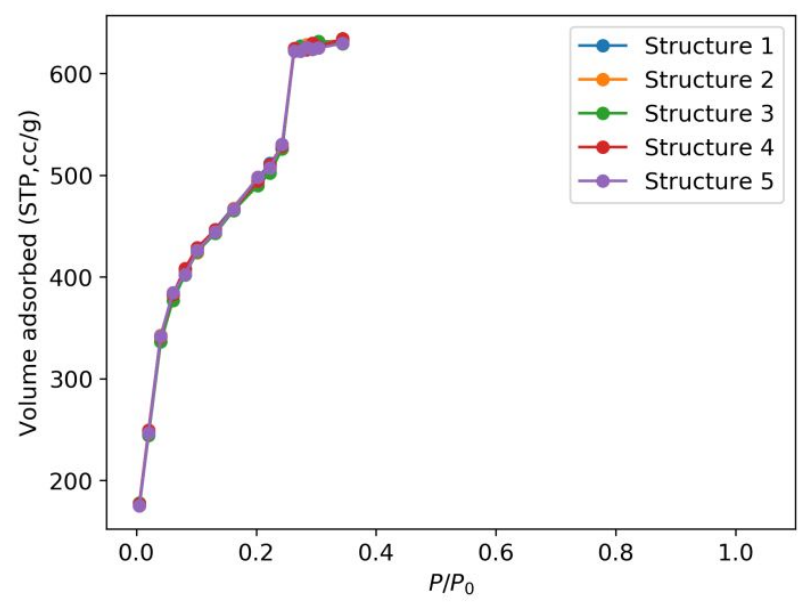

Figure S12: Ar adsorption isotherm for different structures of ZIF-412 that differs only in the relative positioning of linkers. All isotherms are identical.

\section{Algorithm details}

There are 95 different atom types $(40 \mathrm{C}, 33 \mathrm{H}, 15 \mathrm{~N}, 4 \mathrm{O}, 3 \mathrm{Zn})$ in the ZIF-412 cif file ${ }^{9}$. Not all atoms were partially occupied (Figure S8 and Figure S9) — Nitrogen and Oxygen exhibited two partial occupancies (0.7 and 1), carbon existed in occupancies of $0.1,0.3,0.5,0.7,0.9,1$, and all $\mathrm{Zn}$ atoms were fully occupied (Figure S8 and Figure S9).

The structure was divided into 4 groups such that each group contains two linkers out of which one needs to be picked and other to be deleted (Figure S10). Our algorithm deletes or retains atoms within a group to maintain a fixed proportion of linkers within the structure. For example, in group 1, atom types O4, N10, C19A, C18A (and corresponding hydrogens) will be retained with a probability of 0.7 , while atom types $\mathrm{C} 19 \mathrm{~B}$ and $\mathrm{C} 18 \mathrm{~B}$ will be retained with a probability of 0.3 . These two possibilities are mutually exclusive and collectively exhaustive. A specific routine is needed for each group because of different atoms and connectivity. 
F. Comparison of PSD computed from NLDFT and geometric methods

(a)

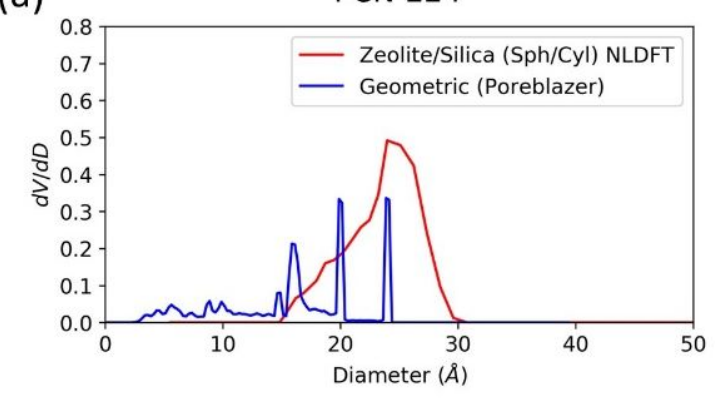

(b)

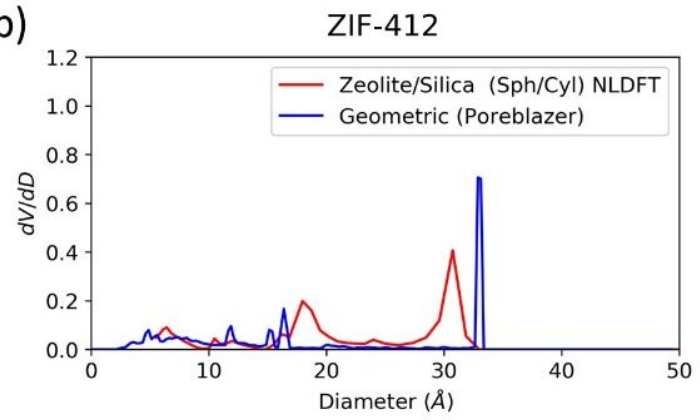

NLDFT fitting comparison in PCN-224

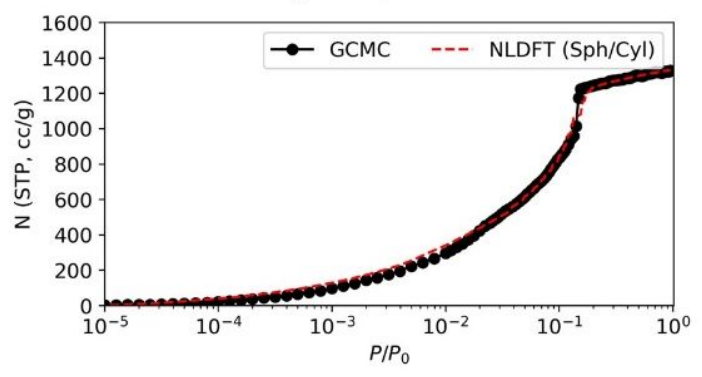

NLDFT fitting comparison in ZIF-412

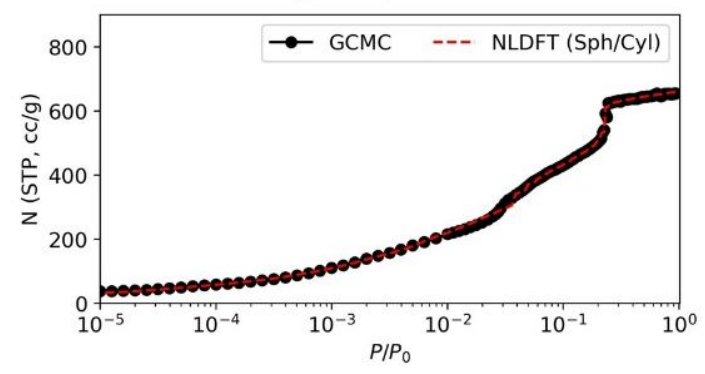

Figure S13: Difference between the PSD computed from the NLDFT method when fitted to the simulated isotherms and Poreblazer ${ }^{11}$ for (a) PCN-224 and (b) ZIF-412.

\section{References}

1. Mayo, S. L.; Olafson, B. D.; Goddard, W. A., Dreiding - a Generic Force-Field for Molecular Simulations. J Phys Chem-Us 1990, 94 (26), 8897-8909.

2. Rappe, A. K.; Casewit, C. J.; Colwell, K. S.; Goddard, W. A.; Skiff, W. M., Uff, a Full PeriodicTable Force-Field for Molecular Mechanics and Molecular-Dynamics Simulations. Journal of the American Chemical Society 1992, 114 (25), 10024-10035.

3. Rowley, L. A.; Nicholson, D.; Parsonage, N. G., Monte Carlo grand canonical ensemble calculation in a gas-liquid transition region for 12-6 Argon. Journal of Computational Physics 1975, $17(4), 401-414$.

4. Potoff, J. J.; Siepmann, J. I., Vapor-liquid equilibria of mixtures containing alkanes, carbon dioxide, and nitrogen. AlChE Journal 2001, 47 (7), 1676-1682.

5. Humphrey, W.; Dalke, A.; Schulten, K., VMD: visual molecular dynamics. J Mol Graph 1996, 14 (1), 33-8, 27-8.

6. Becker, T. M.; Dubbeldam, D.; Lin, L.-C.; Vlugt, T. J. H., Investigating polarization effects 
of CO2 adsorption in MgMOF-74. Journal of Computational Science 2016, 15, 86-94.

7. Shannon, R. D., Dielectric Polarizabilities of lons in Oxides and Fluorides. J Appl Phys 1993, 73 (1), 348-366.

8. Cho, H. S.; Yang, J.; Gong, X.; Zhang, Y. B.; Momma, K.; Weckhuysen, B. M.; Deng, H.; Kang, J. K.; Yaghi, O. M.; Terasaki, O., Isotherms of individual pores by gas adsorption crystallography. Nat Chem 2019, 11 (6), 562-570.

9. Yang, J.; Zhang, Y. B.; Liu, Q.; Trickett, C. A.; Gutierrez-Puebla, E.; Monge, M. A.; Cong, H.; Aldossary, A.; Deng, H.; Yaghi, O. M., Principles of Designing Extra-Large Pore Openings and Cages in Zeolitic Imidazolate Frameworks. J. Am. Chem. Soc. 2017, 139 (18), 6448-6455.

10. Pérez-Pellitero, J.; Amrouche, H.; Siperstein, F. R.; Pirngruber, G.; Nieto-Draghi, C.; Chaplais, G.; Simon-Masseron, A.; Bazer-Bachi, D.; Peralta, D.; Bats, N., Adsorption of CO2, CH4, and N2 on Zeolitic Imidazolate Frameworks: Experiments and Simulations. Chemistry - $A$ European Journal 2010, 16 (5), 1560-1571.

11. Sarkisov, L.; Harrison, A., Computational structure characterisation tools in application to ordered and disordered porous materials. Molecular Simulation 2011, 37 (15), 1248-1257. 